\title{
Tilting Instability and Other Anomalies in the Flux Lattice in Some Magnetic Superconductors
}

\author{
T. K. Ng \\ Department of Physics, HKUST, Kowloon, Hong Kong \\ C. M. Varma \\ Bell Laboratories, Lucent Technologies, Murray Hill, New Jersey 07974
}

(Received 22 November 1996)

\begin{abstract}
The flux-line lattice in the compound $\mathrm{ErNi}_{2} \mathrm{~B}_{2} \mathrm{C}$, which has a tendency to ferromagnetic order in the $a-b$ plane, is studied with the external magnetic field direction close to the $c$ axis. We show the existence of an instability, where the direction of flux lines spontaneously tilts away from that of the applied field as a prelude to the onset of ferromagnetic order and the predicted spontaneous vortex phase. This tilting instability is accompanied by a decreasing longitudinal correlation length of the flux lattice, as observed. [S0031-9007(97)03101-3]
\end{abstract}

PACS numbers: 74.20.De, 74.25.Ha

Recently, it was discovered that the flux-line lattice of the anisotropic magnetic superconductor $\mathrm{ErNi}_{2} \mathrm{~B}_{2} \mathrm{C}$ has very unusual properties. In small angle neutron scattering experiments it was observed that, for applied magnetic field $\vec{H}$ at an angle $\theta_{a} \sim 1.6^{\circ}$ away from the $c$ axis, the average direction of the flux-line lattice is rotated away from the angle of the applied field towards the $a-b$ plane. The angle $\Delta \theta$ between the two increases rapidly at low temperature [1]. The FWHM of the rocking curve $\sigma_{m}$ which measures the longitudinal correlation length $\xi_{L}$ of vortex lines along their length is also found to increase sharply at low temperature, with qualitatively similar temperature dependence as the angle $\Delta \theta[1]$.

It is also observed that $\mathrm{ErNi}_{2} \mathrm{~B}_{2} \mathrm{C}$ has a tendency to develop weak ferromagnetic order at low temperatures $T \leq 2.3 \mathrm{~K}[2,3]$. Such a transition cannot occur with a uniform superconductive state preserved [4-8]. In a previous paper [5] we have suggested that a spontaneous vortex phase occurs in this material in the ferromagnetic state. The magnetic properties of the material can be described by the Ginsburg-Landau (GL) free energy functional [5],

$$
\begin{aligned}
F=\int d^{3} r[ & \frac{1}{2} a|\psi|^{2}+\frac{1}{4} b|\psi|^{4}+\frac{\hbar^{2}}{2 m} \\
& \times\left|\left(\nabla-i \frac{2 e}{\hbar c} \vec{A}\right) \psi\right|^{2}+\frac{\vec{B}^{2}}{8 \pi}+\frac{1}{2} \alpha|\vec{M}|^{2} \\
& \left.+\frac{1}{4} \beta|\vec{M}|^{4}+\frac{1}{2} \gamma^{2}|\nabla \vec{M}|^{2}-\vec{B} \cdot \vec{M}\right],
\end{aligned}
$$

where $\vec{B}=\nabla \times \vec{A}, \vec{M}$ is magnetization, and $\psi$ is the superconducting order parameter. The magnetic component $\vec{M}$ is found to be strongly anisotropic in $\mathrm{ErNi}_{2} \mathrm{~B}_{2} \mathrm{C}$, where magnetization $\vec{M}$ resides essentially only along the inplane easy axis in (100) and (010) directions [2]. We have shown that the unusual in-plane magnetic response of the compound can be explained using the GL functional (1) [4]. In this paper we shall study the out-of-plane magnetic responses of $\mathrm{ErNi}_{2} \mathrm{~B}_{2} \mathrm{C}$ using the same GL functional (1). We shall assume that the magnetization $\vec{M}$ lies only on the $a-b$ plane and shall consider the applied magnetic field making small angle $\theta_{a}$ with the $c$ axis. The in-plane anisotropy of the magnetic component is not included in the GL functional (1) but shall be considered later to understand the square vortex lattice structure.

The competition between magnetism and superconductivity appears in Eq. (1) as a Meissner effect of the superconducting component towards the internal magnetic field produced by the magnetic component $\vec{B}=4 \pi \vec{M}$. For systems with superconducting transition temperature $T_{c}$ higher than the magnetic transition temperature $T_{m}$, the magnetic transition is suppressed. However, the system may go through a second order phase transition to a spiral phase or a first order transition to a spontaneous vortex phase at a slightly lower temperature $T_{s}<T_{m}$ [5-8]. We shall concentrate on the temperature region $T>T_{s(m)}$ in this paper and shall study changes in the magnetic response of the system as $T \rightarrow T_{s(m)}$. In this temperature range, $M$ is small and we can neglect the $M^{4}$ term in the GL functional. The qualitative behavior of the system at this temperature range can be most easily understood by considering the London limit, where $\psi=$ const, and neglecting the $|\nabla \vec{M}|^{2}$ term in $F$ [4].

Writing $\vec{B}=\vec{B}_{c}+\vec{B}_{a b}$, where $\vec{B}_{c}$ and $\vec{B}_{a b}$ are the magnetic field along the $c$ direction and on the $a-b$ plane, respectively, we obtain, after minimizing $F$ with respect to $\vec{M}$ and $\vec{A}, \vec{M}=\vec{B}_{a b} / \alpha$, and $\vec{A}=\lambda_{0}^{2} \nabla \times \vec{B}^{\prime}$, where $\vec{B}_{z}^{\prime}=\vec{B}_{z}$, and $\vec{B}_{a b}^{\prime}=(1-4 \pi / \alpha) \vec{B}_{a b}$. Putting $\vec{M}$ and $\vec{A}$ back into $F$, we obtain

$$
\begin{aligned}
F \sim \int d^{3} r\left\{\frac{-a^{2}}{2 b}+\frac{1}{8 \pi}[\right. & \vec{B}_{z}^{2}+\left(1-\frac{4 \pi}{\alpha}\right) \vec{B}_{a b}^{2} \\
& \left.\left.+\lambda_{0}^{2}\left(\nabla \times \vec{B}^{\prime}\right)^{2}\right]\right\},
\end{aligned}
$$

where $\lambda_{0}^{2}=m c^{2} / 8 \pi e^{2}|\psi|^{2}$ is the London penetration depth for the "pure" superconducting component. $\alpha$ is 
a decreasing function of temperature, and the magnetic transition (in the absence of a superconducting component) occurs at $\alpha\left(T_{m}\right)=4 \pi$. Notice that, for the magnetic field in the $a-b$ plane, the presence of a magnetic component reduces the overall cost in magnetic energy of the pure superconductor by a factor $(1-4 \pi / \alpha)$ and also reduces the London penetration depth from $\lambda_{0}$ to $\lambda=(\sqrt{1-4 \pi / \alpha}) \lambda_{0}[4]$.

To study the magnetic response, we first consider a single vortex line solution in the London limit using Eq. (2). We shall assume that the vortex line is located in the $a-c$ plane and makes a small angle $\theta_{v}$ with the $c$ axis. Minimizing the free energy, we obtain

$$
\lambda_{0}^{2}\left(\nabla \times \nabla \times \vec{B}^{\prime}\right)+\vec{B}=\hat{n} \Phi_{0} \delta(y) \delta\left(x-z \tan \theta_{v}\right),
$$

where $n_{x}=\sin \theta_{v}, n_{y}=0$, and $n_{z}=\cos \theta_{v} . \Phi_{0}$ is the magnetic flux quantum. The equation can be further simplified by using the vector identity $\nabla \times \nabla \times \vec{B}^{\prime}=$ $\nabla\left(\nabla \cdot \vec{B}^{\prime}\right)-\nabla^{2} \vec{B}^{\prime}$ and the Maxwell equation $\nabla \cdot \vec{B}=0$. For small angle $\theta_{v}$, after some algebra and transforming to momentum space, we obtain to order $O\left(\theta_{v}^{2}\right)$,

$$
\begin{aligned}
B_{z}(\vec{q})= & \frac{\left(1-\theta_{v}^{2} / 2\right) \Phi_{0} \delta\left(q_{z}+\theta_{v} q_{x}\right)}{1+\lambda_{0}^{2} q^{2}+\theta_{v}^{2} \lambda^{2} q_{x}^{2}}, \\
B_{x}(\vec{q})= & \frac{\theta_{v} \Phi_{0} \delta\left(q_{z}+\theta_{v} q_{x}\right)}{1+\lambda^{2} q^{2}} \\
& -\frac{4 \pi \lambda_{0}^{2}}{\alpha} \frac{\theta_{v} q_{x}^{2} \Phi_{0} \delta\left(q_{z}+\theta_{v} q_{x}\right)}{\left(1+\lambda^{2} q^{2}\right)\left(1+\lambda_{0}^{2} q^{2}\right)}, \\
B_{y}(\vec{q})= & -\frac{4 \pi \lambda_{0}^{2}}{\alpha} \frac{\theta_{v} q_{x} q_{y} \Phi_{0} \delta\left(q_{z}+\theta_{v} q_{x}\right)}{\left(1+\lambda^{2} q^{2}\right)\left(1+\lambda_{0}^{2} q^{2}\right)} .
\end{aligned}
$$

The various terms in Eq. (4) can be understood as follows: In the absence of the magnetic component $(\alpha \rightarrow$ $\infty$ ), only $B_{z}(\vec{q})$ and the first term in $B_{x}(\vec{q})$ are nonzero and represent the magnetic field of a vortex line tilted away from the $c$ axis on the $a-c$ plane with small angle $\theta_{v}$. In the presence of the magnetic component on the $a-b$ plane, the magnetic response becomes anisotropic, leading to a difference in the penetration depth in $c$ and $a-b$ directions ( $\lambda_{0}$ and $\lambda$, respectively) and a distortion of the vortex core, where a small net magnetization in the $a$ direction is induced. As a result, a small magnetic dipolar field is induced in the $a-b$ plane which is represented by $B_{y}$ and the second term of $B_{x}$. The energy of the single vortex line $\epsilon_{1}$ can be computed using Eqs. (2) and (4). In the limit $(1-4 \pi / \alpha) \ll 1$, we obtain to order $\theta_{v}^{2}$,

$$
\epsilon_{1}=\epsilon_{0}\left(1+a_{1} \theta_{v}^{2}\right)
$$

where

$$
a_{1} \sim \frac{\pi}{\alpha}\left(\frac{\lambda^{2}}{\xi^{2}}-2\right)\left(\ln \frac{\lambda_{0}}{\xi}\right)^{-1}-\frac{4 \pi \lambda^{2}}{\alpha \lambda_{0}^{2}},
$$

where $\xi$ is the superconductor coherence length and $\epsilon_{0} \sim$ $\left(\Phi_{0}^{2} / 4 \pi \lambda_{0}^{2}\right) \ln \left(\lambda_{0} / \xi\right)$ is the vortex line energy when $\theta_{v}=$ 0 . The first term in $a_{1}$ comes from the induced magnetic moment in the vortex core and from the corresponding dipolar field. This term is positive; the other correction terms are negative and represent the lowering in energy from the magnetic component when the magnetic field is in the $a-b$ plane [4]. A similar analysis can also be made when the $|\nabla \vec{M}|^{2}$ term is included in the GL functional. We find that the qualitative behavior of the vortex solution is not modified, except that the divergence in $\lambda^{-1}$ as $T \rightarrow T_{m}$ is removed once the $|\nabla \vec{M}|^{2}$ term is included. In particular, the London penetration depth saturates at a value of order $\lambda \sim\left(\lambda_{0} \xi_{m}\right)^{1 / 2}$ as $T \rightarrow T_{s}$ for transition to the spiral state [4], where $\xi_{m} \sim \gamma^{2} / \alpha$ is the coherence length of the magnetic component.

In the limit $H \sim H_{c 1}$, where the density of vortices is low and interaction between vortices can be neglected, we may study $\theta_{v}$ as a function of the angle of applied field to the $c$ axis, $\theta_{a}$, using Eqs. (5a) and (5b). Consider the Gibb's energy functional,

$$
G=F-\int d^{3} r \frac{\vec{B} \cdot \vec{H}}{4 \pi},
$$

where $\vec{H}$ is the applied field and the total magnetic field $\vec{B}$ is obtained by minimizing $G$ with respect to $\vec{B}$. For small angle $\theta_{a}$ and $\theta_{v}$, Gibb's energy per unit volume is

$$
\frac{G}{V} \sim \frac{B}{\Phi_{0}} \epsilon_{0}\left(1+a_{1} \theta_{v}^{2}\right)-\frac{B H}{4 \pi}\left(1-\frac{\left(\theta_{v}-\theta_{a}\right)^{2}}{2}\right) .
$$

Minimizing $G$ with respect to $\theta_{v}$, we obtain

$$
\theta_{v}=\frac{\theta_{a}}{\left[1+2 a_{1}\left(H_{c 1} / H\right)\right]},
$$

and

$$
H_{c 1}\left(\theta_{a}\right)=H_{c 1}\left(1+\frac{a_{1} \theta_{a}^{2}}{\left(1+2 a_{1}\right)}\right)+O\left(\theta_{a}^{4}\right),
$$

where $H_{c 1}=4 \pi \epsilon_{0} / \Phi_{0}$ is the lower critical field when the external field is along the $c$ axis $\left(\theta_{a}=0\right)$. Notice that $\theta_{v}>\theta_{a}$ when $a_{1}<0$. This may occur when $\lambda / \xi$ is small enough, i.e., when the superconductor is not too strongly type-II and when the system is close to the magnetic instability point $T \rightarrow T_{s}$.

The above analysis can be easily extended to the intermediate density regime $H \sim$ several $H_{c 1}$, where the density of vortices is of order $\left(2 \pi \lambda_{0}^{2}\right)^{-1}$ and the magnetic field $\vec{B}$ is already more or less uniform in the superconductor. This is the regime of experimental interests [1]. The magnetic field $\vec{B}$ in this case can be calculated using Eq. (3), except that the right hand side of the equation is replaced by $\hat{n} \Phi_{0} \sum_{\vec{R}_{n}} \delta\left(y-Y_{n}\right) \delta(x-$ $X_{n}-z \tan \theta_{v}$ ), where $\vec{R}_{n}$ are the positions of the vortices in the vortex lattice. Following procedures similar to above (see also Ref. [9] for details), we obtain in the small $\theta_{a}$ limit,

$$
\begin{aligned}
\frac{G}{V} \sim & \frac{B^{2}}{8 \pi}\left(1-\frac{4 \pi}{\alpha} \theta_{v}^{2}\right)+\frac{B H_{c 1}}{4 \pi}\left(\frac{\ln \left(H_{c 2} / B\right)}{\ln \left(\lambda_{0} / \xi\right)}\right)\left[1+a_{1} \theta_{v}^{2}\right] \\
& -\frac{B H}{4 \pi}\left(1-\frac{\left(\theta_{v}-\theta_{a}\right)^{2}}{2}\right)
\end{aligned}
$$


where $H_{c 2} \sim \Phi_{0} /\left(2 \pi \xi^{2}\right)$ is the upper critical field. Neglecting logarithmic corrections, we obtain, after minimizing $G$ with respect to $B$ and $\theta_{v}, B \sim H-H_{c 1}+B_{0} \theta_{v}^{2}$ and

$$
\theta_{v} \sim \frac{\theta_{a}}{\left(1-\frac{4 \pi}{\alpha}\right)+\frac{H_{c 1}}{H}\left(\frac{4 \pi}{\alpha}+2 a_{1}\right)},
$$

where $B_{o} \sim(4 \pi / \alpha)(1-2 \pi / \alpha) H$ for $H \gg H_{c 1}$ and the angle-dependent magnetization $M\left(\theta_{a}\right)$ is

$$
M\left(\theta_{a}\right)=\frac{B-H}{4 \pi} \sim \frac{B_{0} \theta_{v}^{2}-H_{c 1}}{4 \pi} .
$$

Notice how $\theta_{v}$ is enhanced by the magnetic component in this case. In particular, $\theta_{v}$ is always larger than $\theta_{a}$ for $H \gg H_{c 1}$ and diverges at low temperature $T \leq T_{m}$, in contrast to the low density limit where $\theta_{v}$ may be smaller than $\theta_{a}$ and is larger only when sufficiently close to the magnetic instability. Physically, the instability indicates a magnetic transition where spontaneous magnetization along the $a$ or $b$ directions appears in the system at a low enough temperature, leading to a magnetic-field-assisted spontaneous vortex phase [4], where the vortex lattice tilts spontaneously from the $c$ axis below critical temperature,

$$
T_{\mathrm{sv}} \sim T_{m}-\frac{4 \pi}{\alpha^{\prime}} \frac{H_{c 1}}{H}\left(1+2 a_{1}\right) .
$$

Here $\alpha^{\prime}=d \alpha / d T$. Direct observation of this transition using neutron scattering or magnetic imaging techniques is suggested.

We now consider the case $\theta_{a}=0$ and study thermal fluctuations in positions of vortex lines along the $c$ direction in the intermediate density regime. We consider the model free energy $F_{N}$ for $N$ vortex lines in a sample of thickness $L$, defined by their trajectories $\left[\vec{r}_{j}(z)\right]$ as they traverse through a sample with an external magnetic field along the $z$ axis,

$$
\begin{aligned}
F_{N}= & \frac{1}{2} \epsilon_{t 1} \sum_{j=1}^{N} \int_{0}^{L}\left(\frac{d \vec{r}_{j}(z)}{d z}\right)^{2} d z+\frac{1}{2} \sum_{i \neq j} \int_{0}^{L} \epsilon_{t}\left[\left|\vec{r}_{i}(z)-\vec{r}_{j}(z)\right|\right]\left(\frac{d \vec{r}_{i}(z)}{d z}-\frac{d \vec{r}_{j}(z)}{d z}\right)^{2} d z+\sum_{j=1}^{N} \int_{0}^{L} V_{D}\left[\vec{r}_{j}(z)\right] d z \\
& +\frac{1}{2} \sum_{i \neq j} \int_{0}^{L} V\left[\left|\vec{r}_{i}(z)-\vec{r}_{j}(z)\right|\right] d z
\end{aligned}
$$

where $V_{D}[\vec{r}(z)]$ is a random pinning potential for vortex lines and $V(r) \sim \epsilon_{0} K_{0}\left(r / \lambda_{0}\right)$ is the interaction potential between vortex lines. In the following, we shall replace $V_{D}(\vec{r})$ by the average pinning potential $V_{D}\left[\vec{r}_{j}(z)\right] \sim$ $\kappa_{0}\left(\vec{r}_{j}-\vec{R}_{j}\right)^{2} / 2$, where $\vec{R}_{j}$ are sites on the flux lattice. We shall also expand the interaction potential around its minimum point $V(r) \sim \epsilon_{0}(r / d)^{2}$, where $d \sim\left(\Phi_{0} / B\right)^{1 / 2}$ is the distance between the vortex lattice sites. Note that $d \vec{r}_{j}(z) / d z \sim \theta_{v}\left(\vec{r}_{j}\right)$ in our analysis and the first two terms in Eq. (9) come from terms proportional to $\theta_{v}^{2}$ in the Gibb's energy (7). The tilt modulus $\epsilon_{t 1}$ is chosen such that, when all $\theta_{v}\left(\vec{r}_{j}\right)$ 's are equal, the energy is given by Eq. (7). $\epsilon_{t}(r)$ can be extracted from the free energy $G$ of a flux-line lattice configuration, where all but one of the flux lines have the angle $\theta_{v}\left(\vec{r}_{j}\right)=0$. The field configuration in this case can be calculated from an appropriate combination of Eq. (4) and the field configuration leading to Eq. (7) with $\theta=0$. We shall assume that the flux lines form a square lattice and retain only the nearest neighbor terms in the sum $i \neq j$ in computing $\epsilon_{t}(r)$. The qualitative properties of $F_{N}$ do not depend sensitively on this approximation. We also ignore the ordinary contribution to the tilt moduli expressing the effect of increased line length, as it is negligible compared to the effects we are interested in. We obtain

$$
\epsilon_{t 1} \sim \epsilon_{0}\left[\frac{4 \pi}{\alpha}+2 a_{1}+\left(1-\frac{4 \pi}{\alpha}\right) \frac{H}{H_{c 1}}\right],
$$

where we have neglected the logarithmic correction terms and $\epsilon_{t}(r)=\epsilon_{t 2} \delta(r-d)$, where

$$
\epsilon_{t 2} \sim \frac{\Phi_{0}^{2}}{4 \alpha \lambda_{0}^{2}}\left(\ln \frac{H_{c 2}}{B}\right)\left(\frac{H}{H_{c 1}}-1\right) .
$$

Notice that $\theta_{v} / \theta_{a}=\left(H / H_{c 1}\right)\left(\epsilon_{0} / \epsilon_{t 1}\right)$. Notice also that, at low temperature $T \leq T_{m}, \epsilon_{t 1}$ is a decreasing function of vortex density. In particular, $\epsilon_{t 1} \rightarrow 0$ at the spontaneous vortex phase transition temperature $T \rightarrow T_{\mathrm{sv}}$.

The thermodynamic properties of $F_{N}$ can be obtained rather easily by observing that the variable $z$ can be treated as an imaginary time and the model can be mapped into a quantum mechanical problem of coupled harmonic oscillators on a square lattice with $\hbar \rightarrow T$ [10]. The Hamiltonian of our effective quantum mechanical system is in Fourier space,

$$
\begin{aligned}
H= & \sum_{\mu, \vec{k}} \frac{1}{2 m(\vec{k})} P_{\mu}(\vec{k}) P_{\mu}(-\vec{k}) \\
& +\sum_{\mu, \vec{k}} \frac{m(\vec{k}) \omega(\vec{k})^{2}}{2} X_{\mu}(\vec{k}) X_{\mu}(-\vec{k}),
\end{aligned}
$$

where $\quad X_{\mu}(\vec{k})=N^{-1 / 2} \sum_{i} e^{-i \vec{k} \cdot \vec{R}_{i}} r_{i \mu}, \quad \mu=\hat{x}, \hat{y}, \quad$ and $P_{\mu}(\vec{k})=-i T \partial / \partial X_{\mu}(\vec{k})$ is the canonical momentum conjugate to $X_{\mu}(\vec{k})$. The momentum dependent mass $m(\vec{k})$ and frequency $\omega(\vec{k})$ are

$$
m(\vec{k})=\epsilon_{t 1}+4 \epsilon_{t 2} \gamma(\vec{k}),
$$

and

$$
\omega(\vec{k})=\sqrt{\frac{\kappa_{0}+4 \frac{\epsilon_{0}}{d^{2}} \gamma(\vec{k})}{m(\vec{k})}},
$$


where $\gamma(\vec{k})=\sin ^{2}\left(k_{x} d / 2\right)+\sin ^{2}\left(k_{y} d / 2\right)$. The thermodynamic properties of the flux-line lattice can be obtained easily from (11). We obtain

$$
\left\langle X_{\mu}(\vec{k}, z) X_{\nu}\left(\vec{k}, z^{\prime}\right)\right\rangle=\delta_{\mu \nu} \frac{T}{2 m(\vec{k}) \omega(\vec{k})} e^{-\omega(\vec{k})\left|z-z^{\prime}\right|},
$$

and

$$
\left\langle r^{2}\right\rangle=\frac{1}{N} \sum_{\mu, \vec{k}} \frac{T}{2 m(\vec{k}) \omega(\vec{k})} \sim \frac{T d}{\sqrt{\left(\kappa_{0} d^{2}\right) \epsilon_{t 2}+\epsilon_{0} \epsilon_{t 1}}},
$$

where $\left\langle r^{2}\right\rangle$ is the mean square displacement of flux lines from their equilibrium positions and $\kappa_{0} d^{2} \sim$ average pinning energy of the vortex lines per unit length. Note that $\left\langle r^{2}\right\rangle$ remains finite when $\epsilon_{t 1} \rightarrow 0$ as long as there is a finite pinning strength for the flux-line lattice. The longitudinal correlation length measured in the neutron scattering experiment is $\xi_{L} \sim \omega(\vec{k} \rightarrow 0)^{-1} \sim$ $\sqrt{\epsilon_{t 1} / \kappa_{0}}$, and $\sigma_{m} \sim d / \xi_{L}$, which diverges as $\left(\epsilon_{t 1}\right)^{-1 / 2}$ as the instability towards the spontaneous vortex phase is approached. In particular, we obtain a scaling relation between $\sigma_{m}$ and $\theta_{v} / \theta_{a}$,

$$
\sigma_{m}^{2}\left(\frac{\theta_{a}}{\theta_{v}}\right) \sim\left(\frac{H_{c 1}}{H}\right)\left(\frac{\kappa_{0} d^{2}}{\epsilon_{0}}\right) .
$$

While Eqs. (8a), (8b), and (13) are in good agreement with the existing data in Ref. [1], data over a range of fields and lower temperatures are required to test the theory. Notice that our theory for thermal fluctuations is essentially a Gaussian theory and Eqs. (8a), (8b), and (13) are essentially mean-field results. Deviation from meanfield behavior is expected at temperatures very close to the transition temperature $T_{\mathrm{sv}}$ when the system is in the critical regime. We have estimated the size of the critical regime using the specific heat obtained from our theory, and find that the critical regime is given by

$$
t \leq\left(\frac{\kappa_{0} d^{2}}{\epsilon_{0}}\right)^{1 / 3}\left(\frac{1}{d^{3} \Delta C}\right)^{2 / 3},
$$

where $t=\left|1-T / T_{\mathrm{sv}}\right|$ and $\Delta C$ is the specific heat jump across the transition in mean-field theory. The size of the critical regime is expected to be very small at the intermediate density of vortices $d \sim \lambda_{0}$.

In conclusion, the analysis of this paper and comparison with the results in Ref. [1] strengthen the conclusion arrived at earlier that a spontaneous vortex phase is formed in $\mathrm{ErNi}_{2} \mathrm{~B}_{2} \mathrm{C}$. Neutron scattering experiments or other imaging techniques below $2 \mathrm{~K}$ are urged in order to look for this phase.

We wish to acknowledge very useful discussions with Paul Canfield, Peter Gammel, D. Huse, and U. Yaron. T.K.N. acknowledges partial support by UGC Hong Kong through RGC Grant No. UST623/95P.

[1] U. Yaron et al., Nature (London) 82, 236 (1996).

[2] B. K. Cho et al., Phys. Rev. B 52, 3684 (1995).

[3] P.C. Canfield, S.L. Bud'ko, and B.K. Cho, Physica (Amsterdam) 262C, 249 (1996).

[4] T. K. Ng and C.M. Varma, Phys. Rev. Lett. 78, 330 (1997).

[5] E. I. Blount and C. M. Varma, Phys. Rev. Lett. 42, 1079 (1979).

[6] H. S. Greenside, E. I. Blount, and C. M. Varma, Phys. Rev. Lett. 46, 49 (1981).

[7] M. Tachiki et al., Solid State Commun. 31, 927 (1979); 34, 19 (1980).

[8] C. G. Kuper, M. Revzen, and A. Ron, Phys. Rev. Lett. 44, 1545 (1980).

[9] See, for example, M. Tinkham, Introduction to Superconductivity (McGraw-Hill, New York, 1975).

[10] D. R. Nelson, Phys. Rev. Lett. 60, 1973 (1988); see also D. R. Nelson, in The Vortex State, edited by N. Bontemps, Y. Bruynseraede, G. Deutscher, and A. Kapitulnik (Kluwer Academic, Dordrecht, 1994); G. Blatter et al., Rev. Mod. Phys. 66, 1125 (1994). 